\title{
La construcción de un proceso para la transformación del espacio público: La intervención mural de Boa Mistura en Tetuán, Madrid
}

\section{The construction of a process for the transformation of public space: The mural intervention of Boa Mistura in Tetuán, Madrid}

\author{
AleXandra Delgado-JiméneZ \\ FERNANDO MORAL ANDRÉS \\ ELENA MERINO GÓMEZ \\ Escuela Politécnica Superior y Escuela de Arquitectura. Universidad Antonio de Nebrija, Madrid \\ adelgado@nebrija.es / fmoral@nebrija.es / emerino@nebrija.es
}

Recibido: $20 / 09 / 2017$

Aceptado: 14/01/2018

\section{Resumen}

El espacio público es el lugar de representación de los ciudadanos y su forma determina las conductas y las relaciones sociales que se dan en su seno. Este es el punto de partida del proceso de participación que se ha desarrollado para la transformación de un espacio con carácter de periferia interna en Tetuán, Madrid. Se ha buscado la singularización y la creación de identidad a través de una intervención mural de la mano del colectivo Boa Mistura, que ha jugado el papel de agente mediador entre los intereses públicos y la forma de materializarlos: un arte urbano participativo. El espacio configura la base material de las relaciones y su construcción en un proceso colectivo con agentes de diferente origen y condición (vecinos, niños, migrantes, etc.) supone un ejemplo de la transformación actual del espacio público donde se observa la complejidad creciente en el proceso de toma de decisiones. 


\title{
Palabras clave
}

Espacio público; intervención mural; proceso de participación; arte urbano participativo; Boa Mistura; Madrid.

\begin{abstract}
The public space is the place of representation of the citizens and its form determines the behaviors and social relations that are given within it. This is the starting point of the process of participation that has been developed for the transformation of an area with an internal periphery features in Tetuán, Madrid. Singularization and identity creation have been sought through a mural intervention by the collective Boa Mistura, which has played the role of a mediator between public interests and how to materialize them: a participatory urban art. Space configures the material basis of relationships and their construction in a collective process with agents of different origin and condition (neighbors, children, migrants, etc.) is an example of the current transformation of the public space where we observe the increasing complexity in the process of decision-making.
\end{abstract}

\section{Keywords}

Public space; mural intervention ; participatory process ; participatory urban art, Boa Mistura, Madrid.

Referencia normalizada: DELGADO-JIMÉNEZ, ALEXANDRA - MORAL ANDRÉS, FERNANDO - MERINO GÓMEZ, ELENA (2018): “La construcción de un proceso para la transformación el espacio público: La intervención mural de Boa Mistura en Tetuán, Madrid". Arte y Ciudad. Revista de Investigación, no 13 (abril), págs. 57-82. Madrid. Grupo de Investigación Arte, Arquitectura y Comunicación en la Ciudad Contemporánea, Universidad Complutense de Madrid.

Sumario: 1. El lugar y lo material: la cualificación del espacio público desde la superficie. 2. El proceso: la participación para la transformación del espacio público. 3. La materialización de un proceso para la creación colectiva de un lugar: La intervención mural o "Muro de la convivencia". 4. Conclusiones. 5.- Bibliografía.

* Agradecimientos: A Beatriz López Medina por el impulso y coordinación del proyecto Altěritas en el que se inscribe esta intervención; a Martín Carril Obiols, por ser un referente para el barrio y por su implicación en esta transformación de este espacio público; a Boa Mistura, por hacer realidad los sueños de tantos vecinos 


\section{El lugar y lo material: la cualificación del espacio público desde la su- perficie.}

Es la civilización moderna, esta civilización sin Dios, la que obliga a los hombres a dar tal importancia a la piel. Hoy día no cuenta nada más que la piel. Seguro, tangible, innegable, no hay más que la piel. Es lo único que poseemos. Que sea nuestro. La cosa más mortal del mundo. No hay más que el alma que sea inmortal. Pero, ¿qué cuenta hoy el alma? No hay más que la piel que cuente. Todo es cuestión de piel humana. Nadie se bate ya por la justicia, por la libertad, por el honor. Se bate por la piel, por la asquerosa piel.

Malaparte, 1969 [1949]:74

Las palabras de Curzio Malaparte nos sitúan, de manera cruda, en la importancia que tiene un elemento, aparentemente menor, frente a otros órganos, y que el tiempo continúa avalando como protagonista. Hoy, con un acento mucho más transcendental que en ciertos contextos, lo arma como el valor determinante. Esto sucede en el proyecto de Boa Mistura para el barrio de Tetuán.

Estamos ante un fragmento de ciudad pensado y construido para salvar un desnivel entre dos áreas del barrio de Tetuán. Unas rampas y unas escaleras que acogen entre sus trazas a un mínimo parque de suelo adoquinado y un banco sobre él. En este lugar fueron plantados unos matorrales, en un pequeño espacio estancial aledaño, tres árboles y cuatro cipreses (Fig. 1); estos últimos son los elementos más representativos junto con el muro de contención de hormigón que construye el cambio de niveles. No contamos con superficies considerables para la estancia o el juego, el conjunto está proyectado para ser una vía de comunicación entre alturas muy diferenciadas. El pseudo-parque logra matizar, levemente, esta condición fundacional del sitio. Debemos señalar que estas condiciones físicas no son nuevas en la historia de la conformación de las ciudades. Cuando la topografía ha sido muy abrupta y se ha ocupado por los ciudadanos los sistemas de conexión entre las partes de la urbe siempre han sido puntos complejos y singulares dentro de una trama urbana que, en muchas ocasiones, han sido obviadas dejando que los agentes que intervienen sobre ella adopten una solución de compromiso. Ejemplos como Lisboa o Amman, radicalmente distintos pero marcados por los desniveles, presentan un amplio catálogo de soluciones ante esta problemática, especialmente interesan- 
te en sistemas mecánicos y de escaleras. En este enclave del barrio de Tetuán, compartiendo problemas con las citadas ciudades, no se han materializado sistemas de transferencia entre diferentes cotas de igual calidad y significación que han marcado su carácter de lugar residual en el conjunto de la estructura del barrio. Es cierto que Kevin Lynch afirma que no es posible la existencia razonable de un número desaforado de nodos en la configuración de la ciudad (Lynch, 2012: 95) pero, simultáneamente, señala que las vías habituales y claves de movimientos ciudadanos deben lograr la singularización empleando diferentes recursos formales (Lynch, 2012: 117). La construcción de esa especialización es uno de los retos que el proyecto acomete.

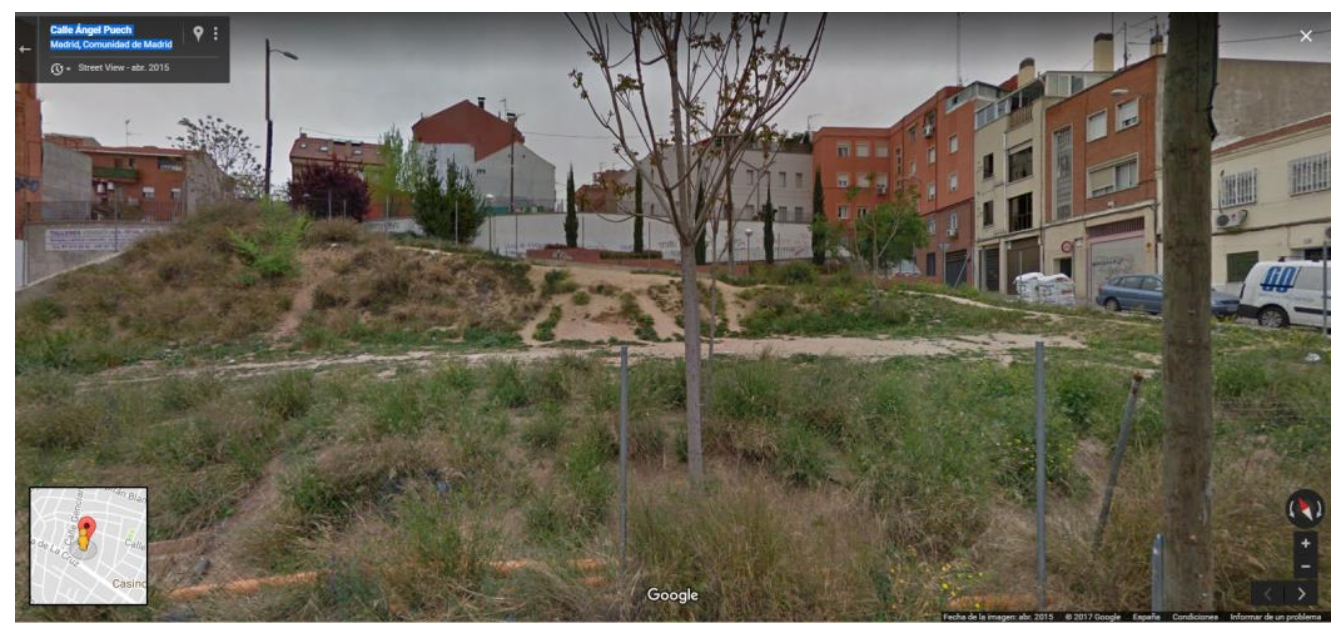

Fig. 1. El muro antes de la intervención. Fuente: Google Street View, abril 2015, https://www.google.es/maps/place/Calle+del+Matadero,+28039+Madrid/@40.4637013,-3.703303,152m/data= !3m1!1e3!4m5!3m4!1s0xd422907fed64e11:0x76c258a3006d943a!8m2!3d40.4636217!4d-3.7026378 Acceso 10 de mayo de 2017.

Nuestro lugar de estudio tiene una doble lectura, minusvalorado en el conjunto de estructuras públicas del barrio pero usado por sus habitantes, de manera profusa. Es una aparente contradicción que apuntala su condición de complejidad, pendiente de un desarrollo que materialice las potencialidades que presenta. Tal y como señala Rem Koolhaas, "donde no hay nada todo es posible. Donde hay arquitectura nada (más) es posible"1 (Koolhaas, 1995: 199).

${ }^{1}$ Citado en Prieto, 2011: 63. 
Es cierto que no es nada ante lo que nos encontramos, pero sí puede verificar la cuestión de encontrarnos ante un mundo de posibilidades.

Podríamos entender que este sitio es un "cuarto espacio" conforme las características que recientemente Patricia Simoes Aelbrecht ha recogido en su investigación; en especial cuando hace referencia al apartado de la "indefinición de forma y función" y al considerar las actividades, nada precisas, que los usuarios desarrollan en este tipo de ámbitos. Los paseantes, los observadores, los que esperan o "pasan el rato" (Simoes, 2016: 11) todos tienen un puesto posible en este espacio desde el que poder potenciar estas actitudes y actividades inciertas que se dan sistemáticamente en la ciudad contemporánea. Es difícil catalogar, desde una sola perspectiva y de una manera ajustada y acertada esta área. La problemática urbana, arquitectónica y social que concurre en él trasciende una catalogación cerrada y definitiva.

Todo lo anterior nos lleva a considerar, desde un enfoque revisado, el concepto de no-lugar como el marco de estudio más interesante desde el que enfrentarnos a los problemas de identidad específica de esta calle-plaza. Augé (1993) considera "los No-lugares" aquellos espacios "impersonales, no apropiables, donde no es posible la alteración pues son espacios de mero tránsito, donde difícilmente se pueden generar sentimientos de pertenencia y relaciones con los otros sujetos que también son transeúntes" (Alguacil, 2008: 204). Manuel Delgado, siguiendo el ideario de Marc Augé caracteriza al "no-lugar como un lugar monótono y frío al que no le corresponde identidad ni memoria y que no tiene nada que ver con contextos espaciales culturalmente identificados e identificadores" (Delgado, 2004: 125). Delgado nos ubica en el contexto intelectual profundo y adecuado para comprender este ámbito. No pertenece a esa pléyade de ámbitos vinculados con el transporte entre metrópolis, tampoco a los que difuminan la ciudad y constituyen la periferia a modo de flecos urbanos, en ocasiones construidos, en ocasiones solares o terrenos abandonados. Pertenece a ese conjunto de espacios urbanos definidos por diferentes razones (funcionales, tecnológicas, económicas, etc.) que no han contemplado el genius loci en su acepción más transcendente. Es un lugar, como tantos otros, sin claridad en sus funciones ni en el ideario que lo soporta (Prieto, 2011: 121), una periferia interna construida con mecanismos (o restos) arquitectónicos sin anclaje sentimental ni social, siendo estos últimos puntos 
determinantes para la comprensión de su estado actual, lejos de ser un punto referencial para el conjunto de sus habitantes.

Este tipo de espacios, esta periferia interna contenida en la ciudad se contrapone con los espacios públicos que son verdaderamente "lugares donde cada uno siente personalmente que los otros pueden, deben, y se apropian del espacio igual que mi persona estableciendo complicidades y relaciones densas" (Alguacil, 2008: 204).

Como se señaló anteriormente cabe intervenir en el sitio buscando una transformación que le conforme desde una perspectiva de ámbito estable y que le aleje de ser un lugar por el que pasar de formas diferentes (Delgado, 2004: 136) y le incruste en una red de espacios públicos matizados por una identidad precisa y una óptica sedentaria que sea singular ante los ojos de los propios y los ajenos (Delgado, 2004: 140). El barrio demanda estos lugares que se asemejen en cualidades y calidad al que existen en otras ubicaciones de la ciudad. La operación planteada es aditiva, no perderá su uso como objeto de comunicación, de tránsito, un valor intrínseco según Augé para estos nolugares, también será un espacio plaza, un lugar para el barrio, en el sentido antropológico del término, capaz de construir relaciones sociales más duraderas y que, en definitiva, mute sus cualidades dinámicas (Prieto, 2011: 109-115) en otras más fijas y transcendentes, nunca accesorias para el colectivo que lo usará. Aldo Rossi señala la asociación que se da entre ciudad y memoria colectiva siendo esta creación humana, la urbe, el punto donde se materializa, a través de diferentes estructuras, la memoria de la población (Rossi, 2004: 226). Estos intangibles gravitan, de manera extremadamente diluida, en esta zona. La intervención del colectivo Boa Mistura nace en esta realidad incompleta del espacio público y no desde una plataforma espectacular.

José Luis Pardo prologa La sociedad del espectáculo de Guy Debord incidiendo en la importancia que para el paisaje urbano y el imaginario colectivo tuvo la conquista de los muros de París por ambiciosas pintadas que explicitaban los valores del Mayo del 68 (Debord, 2010: 9). Las piedras y ladrillos aparejados se transformaron en lienzos ideológicos quebrándose la neutralidad política de ciertos elementos arquitectónicos. La transformación directa de esas edificaciones a través de la pintura rápida, pero conceptualmente densa, fue capaz de construir una nueva y desafiante ciudad en un mínimo periodo de 
tiempo. El valor de la superficie, de la piel de la urbe fue transformado y exacerbado fuera de protocolos administrativos regulares. Esta asimilación del gran valor e impacto en la ciudad, en la arquitectura, no es anecdótico. El tiempo así lo ha reflejado a través de diferentes formalizaciones como la Capilla de los Scrovegni de Giotto, la Majolikahaus de Otto Wagner o más recientemente las intervenciones, en muchos casos anónimas, sobre el Muro de Berlín (Fig. 2) o la mitificación de las actuaciones de Banksy, perfecto conocedor de la mezcla idea, soporte, acción y capaz de influir en importantes procesos políticos (O'Connor, 2017).

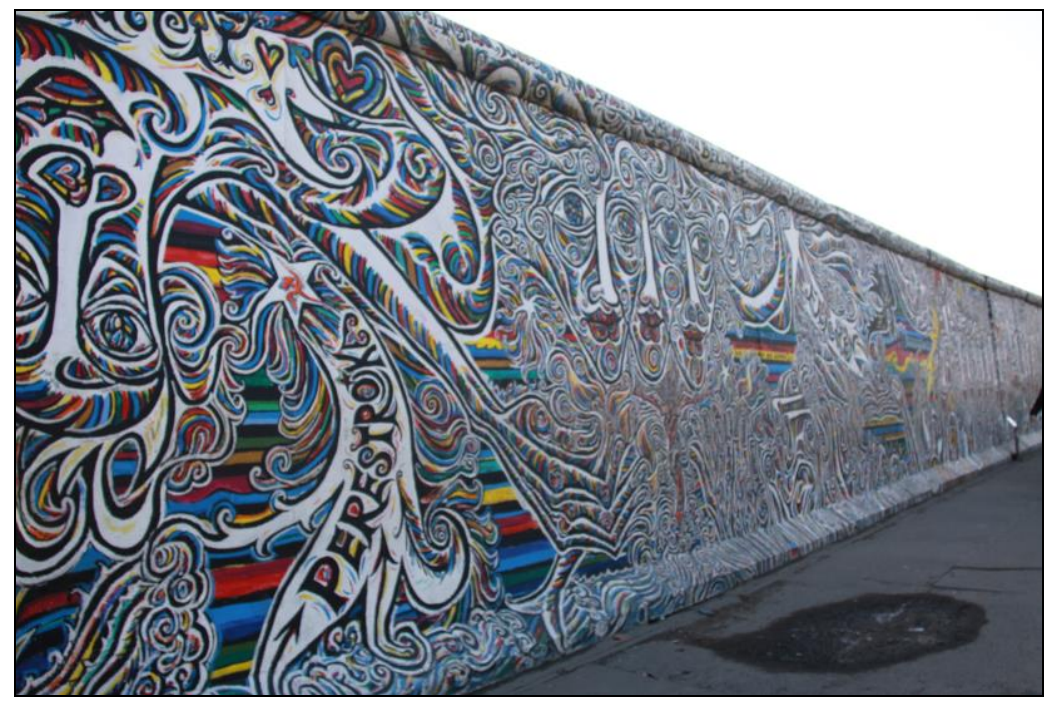

Fig. 2. Pintura en el Muro de Berlín. Fuente: https://laventanadevenus.files.wordpress.com/2013/03/img_8418.jpg Acceso 10 de mayo de 2017.

El valor de la construcción de la fachada, en arquitectura, ha adquirido un protagonismo extremo frente a otros elementos constitutivos de cualquier intervención. No estamos hablando de una cuestión de estricto acabado, también de energía, especialmente en los últimos años, de ecología y, obviamente, de buscar el icono que sea capaz de cambiar un entorno inmediato o de escala mayor. Las doce conferencias en Advanced Building Skins, de alcance internacional, recorren estos puntos desde una fuerte industrialización y mercantilización (Advanced, 2017) pero las pulsiones que habitan la ciudad son mucho más complejas adoptando mecanismos alternativos para las acciones superficiales. 
Kurt Iveson explicita estas realidades a través del concepto "DIY (Do-ItYourself) Urbanism", que pretende enmarcar estas acciones, busca alcanzar, descubrir y confeccionar una nueva ciudad, alternativa a la existente con independencia de encontrarnos en cualquier área de la ciudad (Iveson, 2013: 943). El graffiti, la pintada, alcanza el rango de herramienta fresca y rápida que puede alterar de manera definitiva un área en un espacio temporal exiguo. Es indudable que la pertinencia y calidad de estas acciones siempre ha estado cuestionada pero también debemos considerar cómo en ciertas ocasiones ha sido la manera más accesible que han encontrado quienes viven ese espacio de alterarlo conforme a sus preferencias, de hacerlo más eficiente, en cierto modo, conforme a sus necesidades (Stauskis, 2011: 121). Todas estas obras recobran, en cierto sentido, la importancia que posee la plaza como lugar de concentración de acontecimientos y valores públicos y, sobre todo, como foro democrático de ciudadanos (Giddings, 2011: 203). Boa Mistura es, en gran parte de sus intervenciones, un agente mediador entre esos intereses públicos y la forma de materializarlos. Pueden transformarlos en un acto de resistencia pura como su proyecto Extincta en Higüey (Boa Mistura, 2017a), de recuperación del lugar para los ciudadanos como en Noruega a través del trabajo Dream Hamar (Boa Mistura, 2017a) pero contando, en la mayor parte de sus trabajos, con la apelación a la identidad como base de su trabajo: "el proyecto pretende hacer una intervención paisajística que refuerce el concepto de identidad de la colonia" (descripción del proyecto "Las Américas" en Boa Mistura, 2017) reforzando los valores que pueden unir, emocionalmente, al conjunto de unos ciudadanos al tiempo que implican una transformación efectiva del lugar. Modificando esta retícula al introducir la tipografía (motivo) logramos que se pierda la unidad de vivienda para ganar en concepto de comunidad.

El mensaje busca inspirar diariamente no solo a los vecinos sino también al resto de personas que pasen cerca del edificio y recordarles que cada persona tiene un valor incalculable, independientemente de la realidad del lugar en el que vivan (Descripción del proyecto "Somos Luz" en Boa Mistura, 2017a).

Todo pasa por el lugar, por el descubrimiento profundo de él y por la materialización instantánea empleando la humilde pintura. La intervención superficial ideológicamente sólida y cualitativamente rigurosa puede construir una nueva ciudad lejos del espectáculo denostado por Guy Debord (Debord, 2010: 38) pues en estas intervenciones, como en Tetuán, el diálogo fue la sus- 
tancia que amalgamó toda la intervención. Estamos ante una obra que pretende transformar radicalmente el lugar, haciendo de él un polo de identidad para el conjunto de la sociedad que lo vive (y lo crea) de manera directa. La iconografía vegetal, ligada a ese pasado verde y de agua del territorio sobre el que se asienta la ciudad arma ese puente con el pasado más atractivo y emblemático del área. Complementa e incide en un tema que da nombre a las calles próximas y que enraiza la existencia de un pequeño huerto en las cercanías del emplazamiento trabajado. Todo comparte un ideario, una materia y unas manos que han formalizado ese interés, a través de un mecanismo fácil, abierto y participativo. El lugar, esa periferia interna, ha sido transformado creando un referente necesario y demandado por la comunidad.

\section{El proceso: la participación para la transformación del espacio público.}

La participación es lo que permite la apropiación del espacio público de manera compartida, el sentimiento de ser mío, nuestro, sin excluir de ese sentimiento a los otros ciudadanos, del sentimiento que el espacio urbano también es tuyo o suyo, es lo que hace del espacio público un espacio colectivo.

(Alguacil, 2008: 205)

El espacio público [...] un lugar de relación y de identificación, de contacto entre las gentes, de animación urbana, a veces de expresión comunitaria

(Borja, 2000: 13)

\subsection{Sinergias entre investigación y acción: espacio público y participación.}

La intervención mural objeto de análisis ha supuesto la puesta en práctica de las teorías sobre espacio público y participación que aparecen como una constante en la formación en arquitectura, y más en concreto, en el área de urbanismo, donde ambas cuestiones se plantean siempre asociadas a la transformación del medio urbano, como diría Jordi Borja: “la revalorización del lugar, del espacio público, del ambiente urbano, de la calidad de vida, de la dialéctica barrio-ciudad, del policentrismo de la ciudad moderna"... (Borja, 2000:19).

Resulta de interés comprobar así las verdaderas sinergias que existen entre investigación y acción en cuestiones tan imbricadas como espacio público y participación.

En primer lugar, antes de analizar el proceso que se ha construido para la intervención mural de Boa Mistura en Tetuán, Madrid, y habiendo analizado 
el papel del lugar y lo material, atendiendo a la cualificación del espacio público desde la superficie, se considera la revisión de los conceptos de espacio público y participación y la relación existente entre ambos.

Se parte de un concepto de participación unido al papel de la ciudad como lugar de satisfacción de las necesidades, que conforman por su parte un sistema complejo, y los derechos, entre los que se encuentra el derecho a la ciudad, y más en concreto el derecho al espacio público. La ciudad se considera el espacio para la política y la participación un garante de esta como instrumento para el ejercicio de la política en la ciudad por parte de los ciudadanos. Como indica Alguacil en su artículo clave "Espacio público y espacio político. La ciudad como el lugar para las estrategias de participación":

Considerando que las necesidades son universales e identificables, se muestra cómo la ciudad es el lugar donde mejor se han satisfecho éstas, y como una de ellas, la participación, obtiene un especial significado dado su carácter sinérgico y transversal (Alguacil 2008: 199).

Cabe incidir en la participación como un derecho que permite la intervención en la ciudad, y que por tanto legitima la aspiración de participación como derecho vinculado al derecho a decidir sobre el medio urbano en el que se habita:

En este marco de sujetos provistos de derechos, el concepto de participación se define como la capacidad y derecho de los habitantes/usuarios/ciudadanos de analizar, criticar y transformar el medio en el que viven (Laboratorio Urbano, 2004). Por ello, el derecho de los ciudadanos a ser informados, el derecho a ser consultados y el derecho a tomar parte (Funes Rivas, 2003) alimentarían las legítimas aspiraciones de participación (Delgado Jiménez, 2009).

La participación se convierte en la más transversal de las necesidades humanas y con mayor capacidad sinérgica (Alguacil, 2008: 203). La actividad social sobre el medio ambiente tiene efectos de gran importancia, destacando que

la vinculación de las personas con el medio en el que viven es el primer paso para una reflexión sobre el modelo de desarrollo que estamos produciendo. $Y$ para que se produzca esta vinculación, para desarrollar el sentido de responsabilidad ante el entorno, tanto físico como social y cultural, en el que vivimos, es necesario reforzar los mecanismos de participación en la construcción y transformación de ese medio (Laboratorio Urbano, 2004). 
Si aterrizamos ese medio urbano al espacio público, donde la actividad social y política tiene más representatividad desde sus orígenes, "el espacio público es más público y más colectivo si motiva la participación y menos si inhibe de la misma" (Alguacil, 2008: 205). Se observa entonces la imbricada relación entre participación y espacio público, ya que:

lo conductual determina el espacio físico, el espacio público; y la forma del espacio público determina las conductas y las relaciones sociales. De aquí que la praxis urbana, entendida como la síntesis resultante de la combinación de la acción política (participativa) y el espacio urbano, la podemos considerar como la actividad mediante la cual el sujeto transformador se transforma al participar en la transformación del espacio urbano (Alguacil, Montañés, 2004: 139).

Por tanto, se puede considerar que el hecho de transformar un espacio urbano, un espacio público, puede tener efectos en el vecino/participante que lo transforma.

Si nos centramos en las características del espacio público se debe evitar la definición oficial y administrativa pues esta se centra en cuestiones como la propiedad o una realidad física valorada por la existencia de construcciones, pero que no entra a definir su esencia:

El espacio público es un concepto jurídico: espacio sometido a una regulación específica por pate de la Administración pública, propietaria o que posee la facultad de dominio de suelo y que garantiza su accesibilidad a todos y fija las condiciones de su utilización y de instalación de actividades. El espacio público moderno proviene de la separación formal (legal) entre la propiedad privada urbana (expresada en el catastro y vinculada normalmente al derecho de edificar) y la propiedad pública (o dominio público por subrogación normativa o por adquisición de derecho mediante cesión) que normalmente supone reservar este suelo libre de construcciones (excepto equipamientos colectivos y servicios públicos) y cuyo destino son usos sociales característicos de la vida urbana (esparcimiento, actos colectivos, movilidad, actividades culturales y a veces comerciales, referentes simbólicos monumentales, etc.) (Borja, 2000:13).

Si nos centrarnos en su realidad física y social, se debe partir del espacio público como un espacio complejo y político, donde se construye la identidad:

un espacio complejo (variado, plural, de mezcla) y a la vez sencillo (percibido, controlado, legible, simbólico...) [...]el espacio público como espacio político debe orientarse a construir una identidad de identidades que englobe a todas, a la misma vez que defiende a cada una de ellas (Alguacil, 2008: 206 y ss.). 
Siguiendo esa línea, "la calidad del espacio público se podrá evaluar sobre todo por la intensidad y la calidad de las relaciones sociales que facilita, [...[ y por su capacidad de estimular la identificación simbólica, la expresión y la integración culturales" (Borja, 2000:14). Lógicamente, esa calidad del espacio público debe estar asociada a una base material que permita las relaciones y la identidad e identificación, entre otras cuestiones, y en ese aspecto es en el que se ha incidido con la intervención mural que se analiza.

A modo de resumen de lo anteriormente reseñado, se recogen las necesidades aplicadas a la ciudad y el espacio público (Tabla 1), en las que interviene de manera destacada la participación, por su carácter transversal, así como aquellas relacionadas con la intervención mural: creación, recreo e identidad.

\begin{tabular}{|l|l|l|l|l|}
\hline $\begin{array}{l}\text { Categoría } \\
\text { Axiológica }\end{array}$ & \multicolumn{1}{|c|}{$\begin{array}{c}\text { Ser (sujeto de } \\
\text { derechos) }\end{array}$} & \multicolumn{1}{|c|}{ Tener } & \multicolumn{1}{|c|}{ Hacer } & \multicolumn{1}{c|}{ Relaciones } \\
\hline $\begin{array}{l}\text { PARTICI- } \\
\text { PACIÓN }\end{array}$ & $\begin{array}{l}\text { Derechos de Ciu- } \\
\text { dadanía } \\
\text { Derecho al buen } \\
\text { gobierno } \\
\text { Derecho a la pro- } \\
\text { ducción del espa- } \\
\text { cio }\end{array}$ & $\begin{array}{l}\text { Responsabilidad } \\
\text { Capacidad de } \\
\text { decisión } \\
\text { Capacidad de } \\
\text { gestión }\end{array}$ & $\begin{array}{l}\text { Compartir } \\
\text { Decidir } \\
\text { Asociarse }\end{array}$ & $\begin{array}{l}\text { De reciprocidad, } \\
\text { consenso, } \\
\text { negociación, } \\
\text { co-responsabilidad }\end{array}$ \\
\hline $\begin{array}{l}\text { CREA- } \\
\text { CIÓN }\end{array}$ & $\begin{array}{l}\text { Derecho al trabajo } \\
\text { y la formación }\end{array}$ & $\begin{array}{l}\text { Formación } \\
\text { Información } \\
\text { Soportes, recur- } \\
\text { sos, equipamien- } \\
\text { tos polivalentes }\end{array}$ & $\begin{array}{l}\text { Analizar } \\
\text { Diseñar } \\
\text { Planificar } \\
\text { Innovar }\end{array}$ & $\begin{array}{l}\text { De colaboración } \\
\text { De cooperación }\end{array}$ \\
\hline RECREO & $\begin{array}{l}\text { Derecho al tiempo } \\
\text { libre. } \\
\text { Derecho a la libre } \\
\text { circulación y al } \\
\text { espacio público }\end{array}$ & $\begin{array}{l}\text { Espacios libres, } \\
\text { Tiempo liberado, } \\
\text { Equipamientos }\end{array}$ & $\begin{array}{l}\text { Imaginar, } \\
\text { crear }\end{array}$ & $\begin{array}{l}\text { De proximidad, amis- } \\
\text { tad, vecindad, familia }\end{array}$ \\
\hline $\begin{array}{l}\text { Derechos cultura- } \\
\text { les }\end{array}$ & $\begin{array}{l}\text { Autoestima } \\
\text { Ámbitos de per- } \\
\text { tenencia } \\
\text { Espacios simbóli- } \\
\text { cos apropiados }\end{array}$ & $\begin{array}{l}\text { Apropiarse } \\
\text { Participar } \\
\text { Afirmarse } \\
\text { Tolerarse }\end{array}$ & $\begin{array}{l}\text { Múltiples con los } \\
\text { otros } \\
\text { Interculturalidad }\end{array}$ \\
\hline
\end{tabular}

Tabla 1. Selección de temas de la matriz de necesidades aplicadas al satisfactor ciudad y espacio público a partir de (Alguacil 2008: 202) y a su vez (Max-Neef, Elizalde y Hopenhayn 1986: 42). 


\subsection{El proceso de implicación de agentes: la creación de un cuerpo colectivo.}

El proceso "para la transformación del espacio público a través de una intervención mural en Tetuán" se inició con el proyecto Altěritas, concedido a la Fundación Nebrija por la Consejería de Políticas Sociales y Familia de la Comunidad Autónoma de Madrid. Se trata de una iniciativa que coordina el Departamento de Lenguas Aplicadas y Educación de la Universidad Nebrija que sigue la línea de actividades de sensibilización y formación a la comunidad inmigrante de gran tradición en la universidad, como es el caso del Diploma Letra (Universidad Nebrija, 2016a). El proyecto Altěritas versa sobre los diálogos y representaciones sobre alteridad y descubrimiento intercultural, y tiene como objetivo:

Orientar las actividades de extensión universitaria de la Universidad Nebrija a la reflexión sobre la alteridad, el diálogo entre culturas y el descubrimiento de los universales compartidos, mediante la invitación a participar de manera conjunta a los estudiantes y a los inmigrantes interesados en compartir sus vivencias y creaciones, promoviendo la difusión pública de las mismas (Departamento de Lenguas Aplicadas y Educación de la Universidad Nebrija, 2016).

En el proyecto se plantean numerosas actividades como conferencias, talleres o torneos de fútbol, entre otras, focalizadas en el diálogo entre comunidad universitaria e inmigrante. Supone el punto de partida y permite la financiación mínima que este tipo de proyectos necesita.

Se plantea utilizar la posibilidad que sirve el proyecto Altěritas, con el objetivo de proponer una intervención para crear un lugar de encuentro entre la comunidad universitaria, vecinos y migrantes, utilizando la pintura como instrumento de integración social. La elección del distrito de Tetuán no es casual, sino que está en el corazón del proyecto. Este distrito cercano al Campus de Dehesa de la Villa de la Universidad, ha sido objeto de análisis e intervención teórica en el marco de la asignatura iniciática de Urbanismo, en el grado de Arquitectura, durante varios cursos. En este caso se busca establecer sinergias entre investigación, docencia y acción, relacionando dos cuestiones cruciales como son la participación y el espacio público. En una cultura de "usar y tirar" se trata aquí de lo contrario, la mejora de un espacio a través de la participación, la creación artística, el recreo, el arte urbano participativo y la mejora de la identidad. Un desarrollo personal y social para apropiarse de un espacio público que no tiene todavía carta de naturaleza como tal. 
Este es el punto de partida para la construcción de un proceso de participación colectivo con la configuración de un espacio en común entre agentes de diferente origen y condición: vecinos, niños, migrantes, estudiantes universitarios, profesores, agentes sociales, artistas y representantes públicos. Se inician las primeras gestiones a finales de 2015 y se contacta con una persona clave en el proceso como es Martín Carril Obiols, que coordina la Huerta de Tetuán, situada en el barrio de Valdeacederas, del distrito de Tetuán de Madrid, con la que se establece una alianza. Esta huerta urbana está situada en el solar de la calle Matadero, en la trasera del colegio Juan Ramón Jiménez. Se creó en el año 2013:

el huerto urbano, con intervenciones de tipo artístico, dentro del programa "Mejora del Paisaje Urbano". En el caso concreto de la Huerta de Tetuán, varios colectivos y agentes culturales, además de artistas invitados, están trabajando en la elaboración de un huerto urbano (a cargo del colectivo Moenia), un cerramiento experimental con palés (del grupo la Fresquera) y del proyecto Emergiendo (de Mood Studio Fotográfico y la Galería Magdalena, con la colaboración de decenas de vecinos de Tetuán (La aventura de aprender).

La relación con Martín Carril y otros vecinos que gestionan la Huerta de Tetuán, permiten el intercambio con los estudiantes de Arquitectura y la búsqueda de un lugar en el que intervenir por parte de los vecinos y teniendo en mente Boa Mistura, el colectivo de artistas conocido internacionalmente y uno de los máximos exponentes de arte urbano participativo en nuestro país (Boa Mistura, 2017b). El diálogo sobre la elección de una pintura mural es fructífero, ya que los vecinos implicados consideran que es muy positivo por su valor artístico y por ser un tipo de arte urbano participativo, y por tanto, pueden intervenir un gran número de vecinos y, en general, de perfiles, como en este caso migrantes y miembros de la comunidad universitaria, entre otros.

Se consigue la participación de Boa Mistura, con una implicación máxima desde el primer momento, previendo las fechas de intervención en junio de 2016. Están abiertos a los diferentes lugares de intervención que se barajan inicialmente, esperando a aportar propuestas artísticas alternativas cuando se fije el espacio de intervención y los vecinos hayan dado su opinión sobre los deseos y objetivos respecto a la pintura mural.

Visitan a los alumnos de urbanismo en mayo de 2016, concretamente los artistas Javier Serrano y Pablo Ferreiro (Fig. 5) para tener una primera toma de contacto con los estudiantes y que conocieran en qué consisten sus proyectos y 
cómo se desarrollan, y la filosofía detrás de esta intervención en la que los alumnos iban a formar parte:

Según explicaron, hay un antes y un después en los lugares en donde Boa Mistura ha desarrollado un proyecto. Porque "la pintura empieza a tejer unas relaciones entre sí", aseguró Javier Serrano. Además, Pablo Ferreiro señaló que el colectivo intenta vincular todos los proyectos con los espacios en los que se van a llevar a cabo. "Su fortaleza es la comunidad porque son los vecinos los que empiezan a construir. Buscamos esa identidad y paisaje propio", añadió. (Universidad Nebrija, 2016b).

Finalmente el interés de los vecinos de la Huerta de Tetuán, se centra en el muro situado entre las calles Matadero y Ángel Puech², justo al lado de su huerta. Se trata de un muro realizado para salvar el desnivel, de reciente construcción, como se observa porque en la ortofotografía aérea de 2007 (Fig. 3) no estaba ejecutado todavía (Fig. 4).

Se realizan varias reuniones con diferentes áreas del Ayuntamiento de Madrid hasta conseguir finalmente el apoyo del Área de Paisaje Urbano del Ayuntamiento de Madrid y la de la Junta de Distrito de Tetuán, ya que es pertinente la autorización para la actuación.

Es positivo reseñar las orientaciones estéticas del Área de Paisaje Urbano, para la integración de la pintura mural, así como al hecho de que materialmente el muro pudiera intervenirse sin la necesidad de un andamiaje, lo que supondría por una parte, otros trámites y gestiones, y que, por otra parte, impediría lógicamente la participación más directa de todo tipo de público.

Una vez conocido el lugar y fijado el calendario, del 27 de junio al 1 de julio de 2016, se empezó a hacer un cuestionario a los vecinos de la Huerta de Tetuán sobre los motivos de diseño para esta localización. Además se contó con la colaboración de "Aprende tu barrio" con acciones encaminadas a que los niños participen y aporten ideas para mejorar la ciudad, cobrando en la pintura del muro un papel protagonista. En el colegio próximo de Juan Ramón Jiménez se trabajó también en los motivos que se deseaban dibujar en el muro.

2 Localización: https://www.google.es/maps/place/Calle+del+Matadero,+28039+Madrid/@40. 4637013,-3.703303,152m/data=!3m1!1e3!4m5!3m4!1s0xd422907fed64e11:0x76c258a3006d943a!8 $\mathrm{m} 2 ! 3 \mathrm{~d} 40.4636217 ! 4 \mathrm{~d}-3.7026378$ 


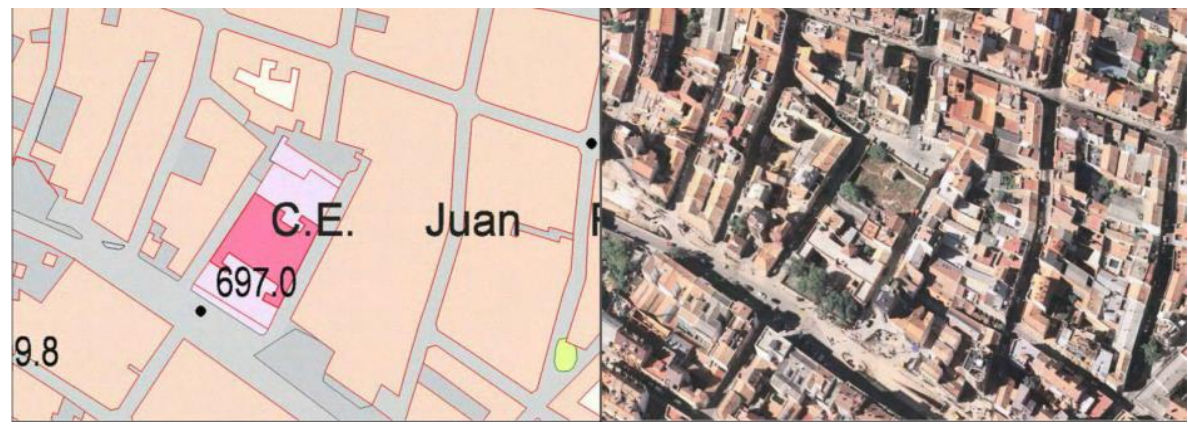

Fig. 3. Planimetría y ortofotografía aérea del entorno del muro objeto de intervención, 2007. Fuente: Foto histórica 2007 - Dirección General de Urbanismo Consejería de Medio Ambiente, Administración Local y Ordenación del Territorio, Zonas urbanas y entorno 2007, http://www.madrid.org/cartografia/ planea/cartografia/html/web/Visor2Vistas.htm\# Acceso 10 de mayo de 2017.

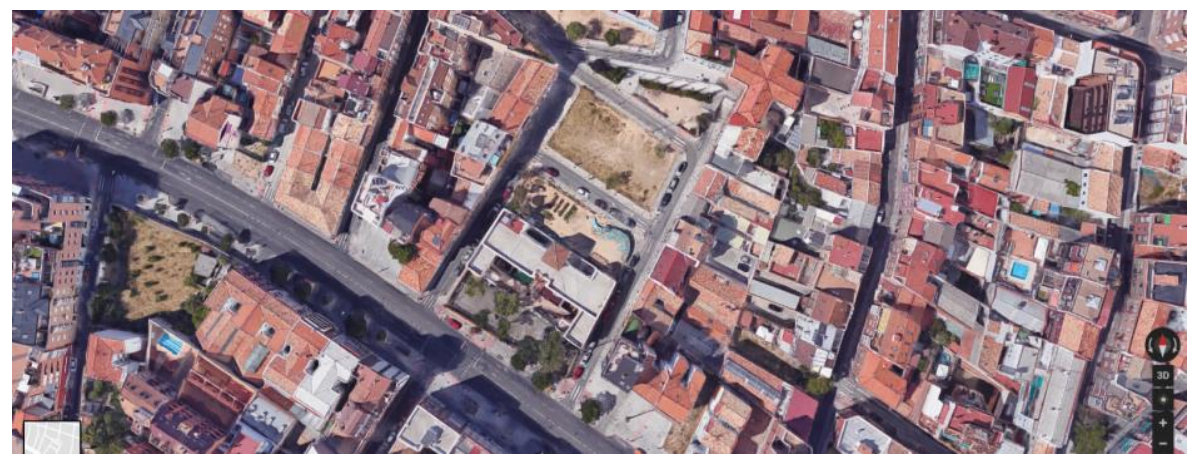

Fig. 4. Ortofotografía aérea del entorno del muro objeto de intervención, 2017. Fuente: Google Maps, 2017.

Boa Mistura presentó varias versiones y finalmente quedó fijada una. Todas las opciones estaban basadas en las ideas que se habían recopilado por parte de vecinos y niños, así como de las visitas que el colectivo había hecho al muro en el que se iba a intervenir. La semana de autos se realizó la presentación de la propuesta mural definitiva en la Universidad Nebrija, junto al grupo de estudiantes que iban a trabajar diariamente en la intervención y que iban a su vez a formar a las decenas de personas que habían indicado que participarían. Y, por último, y desde el primer momento, como hilo conductor de toda la construcción del proceso, como la argamasa de lo que se pretende edificar se encuentra la implicación de un gran número de vecinos, inmigrantes, alumnos universitarios y profesores. 
A través de la Huerta de Tetuán se fue conociendo la opinión de los vecinos y ellos mismos también gestionaron la participación de los niños (finalmente más de cien niños participaron con monitores). Asimismo, se fue contactando desde el Departamento de Lenguas Aplicadas y Educación con estudiantes de español como lengua extranjera, colectivos y asociaciones de inmigrantes así como asociaciones de integración psicosocial, que acudieron también en grupos coordinados, para que el flujo de pintores fuera coherente en cada momento y hubiera trabajo para todos.

Fig. 5. Javier Serrano y Pablo Ferreiro de Boa Mistura con los alumnos de Urbanismo, 2016 (Fuente: Universidad Nebrija).

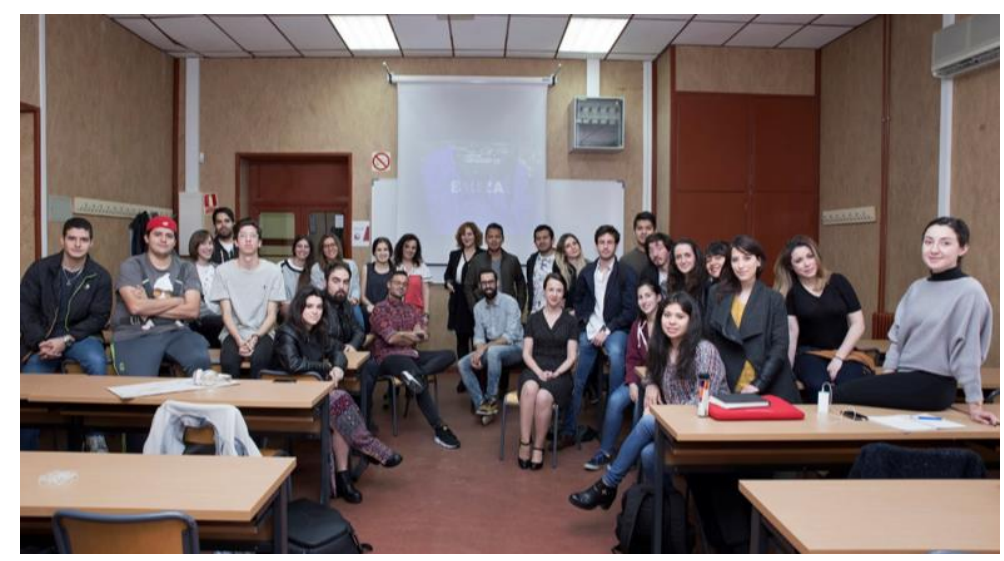

En la semana de la intervención mural, la participación de los vecinos, migrantes, niños fue muy alta respecto al tamaño de la intervención, con más de ciento cincuenta "pintores" entre todos los colectivos anteriormente mencionados, reflejo del interés retroalimentado en todo el proceso.

Por último, y como resumen rápido para lo dilatado del proceso (unos nueve meses) respecto al tamaño de la intervención, se encuentra esta noticia destacada en portada del periódico de barrio:

La Universidad Nebrija y el colectivo Boa Mistura realizan una intervención urbana en el distrito de Tetuán en el que participan niños y vecinos del barrio. Comenzó siendo una idea con el objetivo de reflexionar sobre la alteridad y el diálogo entre culturas y poco a poco el proyecto Alterritas ha ido tomando forma: encuentros, conferencias y campeonatos de fútbol, entre otras actividades, en donde estudiantes e inmigrantes han tenido un espacio de encuentro para compartir vivencias.

[...] Hace unos días, Boa Mistura ideó y participó en el "Muro de la convivencia", una amplia pared situada al final de la calle del Matadero, junto a la 
Huerta de Tetuán. La iniciativa está promovida por la Universidad Antonio de Nebrija, dentro del proyecto Alteritas, cuyo objetivo es impulsar actividades en las que participen de manera conjunta estudiantes y la comunidad inmigrante. El Área de Paisaje Urbano del Ayuntamiento, y la Huerta de Tetuán, con la coordinación de Martín Carril, también han hecho posible esta acción, que ha contado además con la participación de vecinos del barrio y de hasta 70 niños de diferentes colectivos. [...]

En esta acción participa también el proyecto "Aprende tu barrio", que ya ha organizado "fiestas de mapeo" de la accesibilidad en varias zonas del distrito, o ha puesto en marcha iniciativas como "Madrid también es nuestra", encaminada a que los niños participen y aporten ideas para mejorar la ciudad (Tetuán 30 días, 2016: 7).

\section{La materialización de un proceso para la creación de un lugar: la inter- vención mural o "Muro de la convivencia".}

Nosotros también tenemos derecho a la belleza (Una abuela de favela, en Sao Paulo - Brasil)

Borja, 2000:24

\subsection{La pintura como representación del barrio.}

Uno de los puntos de partida para fijar el diseño del mural era que la pintura representara al barrio. Asociado a esto estaba la indicación de que no se utilizara el texto, ya que éste había sido utilizado profusamente en la acción "Paisaje Tetuán", llevada a cabo en todo el distrito solo tres años antes de la intervención. Es por eso que aunque en los trabajos de Boa Mistura el verso y la palabra suelen ser el hilo conductor, en este caso se dejaron a un lado para centrarse en dos cuestiones que reflejan la nueva identidad que quiere darse a este espacio: los motivos vegetales y los colores asociados a estos y a la historia del barrio (Fig. 6).

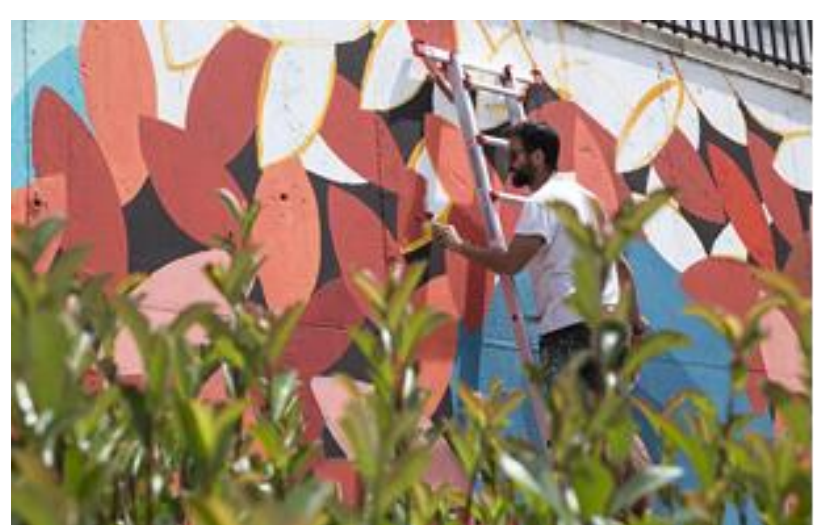

Fig. 6. Pablo Purón, del colectivo Boa Mistura, ilustrando el muro con motivos vegetales, 2016 (Fuente: Universidad Nebrija). 
Como indica Javier Serrano, uno de los miembros de Boa Mistura: "hemos aprovechado las ideas que nos han transmitido los vecinos, principalmente motivos vegetales, que hacen referencia a las calles cercanas, como Cactus, Miosotis, Aligustre, Alfalfa..." (Tetuán 30 días, 2016: 7).

Por otra parte, la selección de colores se ha centrado en los colores de la vegetación, las flores teniendo en cuenta además la estrecha relación del barrio con el agua:

Así, el mural sería como "la pared" que cierra la panorámica de la Huerta, "de hecho los colores verdes empleados hacen juego con sus tonos, mientras que los azules homenajean al agua", otro elemento de la zona, representado por el Canal Bajo (Fig. 7, 8 y 9) (Tetuán 30 días, 2016:7).

Fig. 7. Materiales para los trabajos de pintura mural, al fondo la Huerta de Tetuán, 2016.

Figs 8 y 9. "El Muro de la convivencia”, durante la realización y finalizado.

(Fuente: Universidad Nebrija).
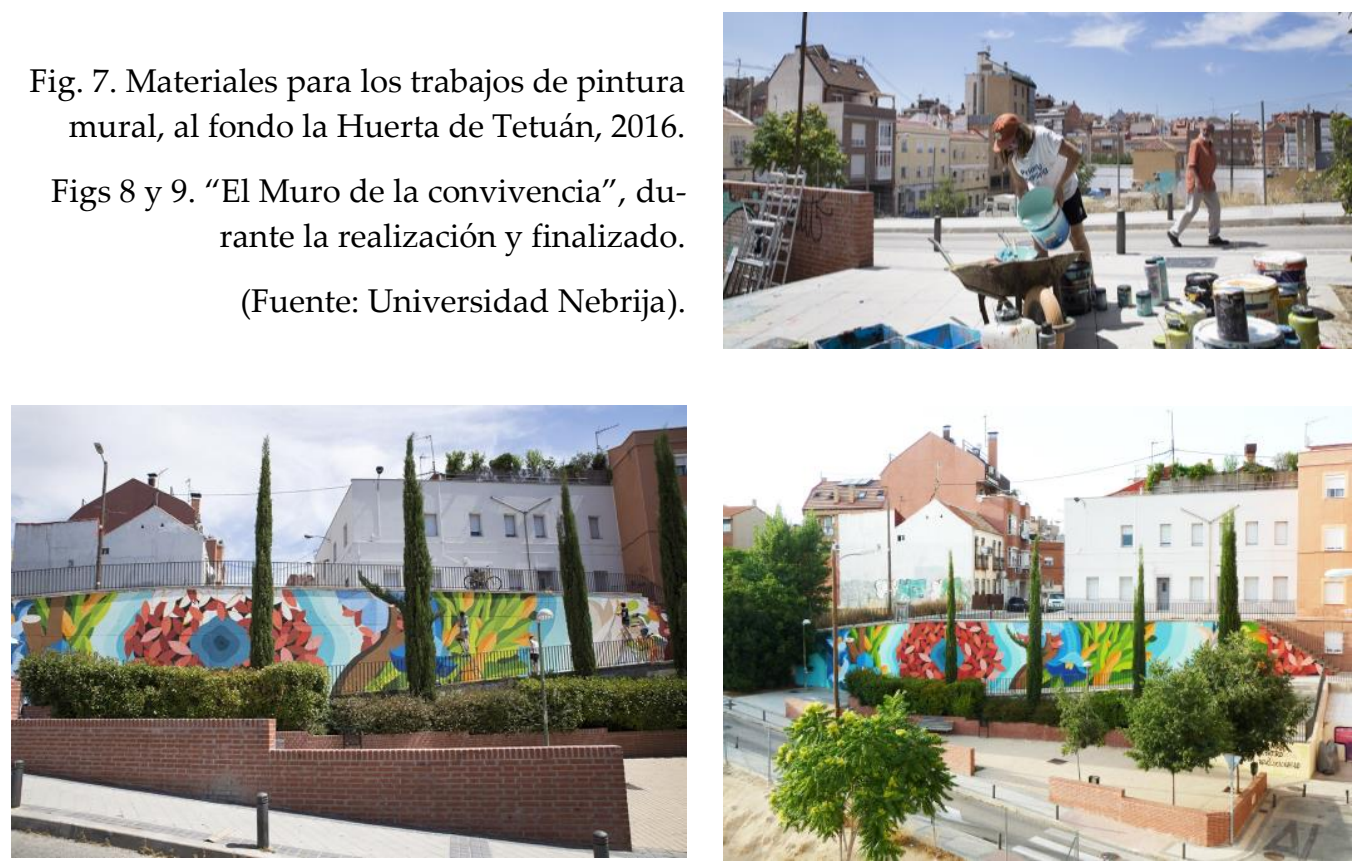

El diseño se define entonces por la observación del entorno y la complementariedad con la Huerta de Tetuán lo que hace que los motivos de hojas y flores inunden todo el muro.

El azul del agua también es el hilo conductor de este jardín vertical que completa como una "cuarta pared" la Huerta de Tetuán. 


\subsection{Difusión en medios y acogida posterior.}

La difusión en medios ha sido intensa pero centrada en medios más locales o con implicación directa. Principalmente se han centrado en el periódico local Tetuán 30 días, en la emisora de Radio 3 de RNE (Radio Nacional de España), con una entrevista a uno de los artistas del colectivo Boa Mistura y a través de las noticias web de la Universidad Nebrija. También se ha incorporado la información sobre la intervención dentro del dossier de Alterritas, ya que como se ha indicado anteriormente, estaba concedido por la Comunidad de Madrid. Se reseña a continuación la información difundida por la Universidad Nebrija:

Un espacio que culmina esta semana con la intervención urbana en el distrito de Tetuán, entre las calles Matadero y Ángel Puech, en el barrio de Valdeacederas ${ }^{3}$. Allí, estudiantes y profesores de Universidad Nebrija y los miembros del colectivo Boa Mistura han realizado un mural en uno de los muros de la zona. Para llevar a cabo este proyecto, han contado con la colaboración de los vecinos del barrio, entre ellos, más de 100 niños que han querido participar en la pintada. [...]

Para conocer todos los detalles de este proyecto, Radio 3 se acercó al barrio de Tetuán, en donde entrevistó a uno de los chicos del colectivo Boa Mistura (Fig. 10). La entrevista se podrá escuchar este viernes 1 de julio o el lunes 4 de julio entre las 9.00 y las 11.00 en difusión nacional (Universidad Nebrija, 2016a).

Y para conocer la acogida por parte del medio local Tetuán 30 días, se destacan unas líneas que muestran el entusiasmo de este medio respecto a la intervención mural del colectivo de artistas Boa Mistura:

Faltaron en el proyecto "Paisaje Tetuán", pero el distrito ya se ha quitado la espina clavada de prestar uno de sus muros para el colectivo de arte urbano de moda dentro y fuera de España (Tetuán 30 días, 2016: 7).

En cuanto a la acogida posterior, cabe destacarse que solo unos pocos meses después de haberse acabado la pintura mural, han aparecido pintadas, lo que se puede considerar negativo, ya que puede entenderse como un desprecio al trabajo de todo un barrio. Pero en todo caso, las pinturas murales, como

\footnotetext{
3 https:/www.google.es/maps/place/Calle+del+Matadero,+28039+Madrid/@40.4637013,-3.703303, 152m/data=!3m1!1e3!4m5!3m4!1s0xd422907fed64e11:0x76c258a3006d943a!8m2!3d40.4636217!4d3.7026378
} 
obras de arte urbano participativo, siempre están expuestas a posteriores intervenciones o vandalismo, ya que son obras de arte "vivo" desde el punto de vista de que están abiertas al cambio por estas acciones, pero también por las condiciones atmosféricas a las que están expuestas.

En caso de que la intervención mural arraigue como propia dentro del corazón del barrio de Valdeacederas, en Tetuán, se debería mantener por parte de los vecinos, para poder conservarse. Si no es así, se convertirá por lo menos en símbolo de que durante unos meses de proceso, y unos días de pintada, el barrio se abrió a otros, viéndose complementado y puesto en valor por la "alteridad".

Fig. 10. Entrevista de Radio 3 a un miembro del colectivo Boa Mistura, Pablo Purón, durante la realización de la pintura mural, 2016. Fuente: Universidad Nebrija.

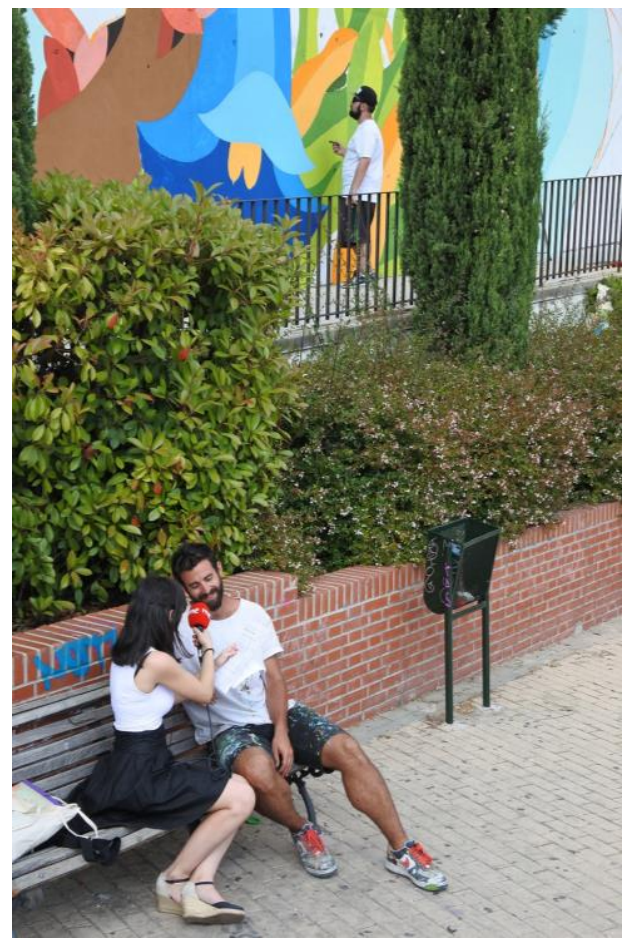

\section{Conclusiones.}

Una intervención de arte urbano participativo supone la revalorización de un lugar a través de lo material, pero también de un proceso, porque sin participación no pueden conseguirse los objetivos de creación, recreo e identidad que tienen asociadas este tipo de obras.

La construcción de un proceso de participación supone la puesta en valor del derecho de producción del espacio, la creación de consensos y la gestión para la involucración de agentes diversos. Desde el punto de vista de la intervención mural, la participación se da a través de la creación artística compartida con la participación de diferentes perfiles, y la asociación de esta inter- 
vención a otras que se han dado cercanas en el tiempo y en el espacio como las desarrolladas en la Huerta de Tetuán y, en general, en Paisaje Tetuán.

La creación es otra cuestión de interés para este tipo de procesos, ya que el diseño no es libre, la manida "libertad del creador" sino que se trata de un diseño teniendo en cuenta las propuestas de vecinos, niños y la administración pública, lo que para el proceso son los requerimientos. En cuanto a la propia intervención la creación está mediada por las indicaciones durante el proceso de pintura por parte del colectivo de artistas Boa Mistura, y la cooperación entre el grupo de colaboradores que participaron a diario en todo el proceso, y aquellos que lo hicieron esporádicamente.

En cuanto al concepto clave de recreo, se considera que la síntesis es ser una obra de arte urbano participativo, donde la mejora del espacio público se hace a través del embellecimiento, con una propuesta creativa de proximidad. No es una obra destinada al museo, sino a generar un lugar donde solo existe un espacio residual.

Y respecto a los aspectos de identidad, en la construcción del proceso se ha podido observar una mejora de la autoestima de los vecinos y de todos los participantes ("me involucro", "saco algo adelante", "mejoro mi entorno", "me tienen en cuenta", etc.); la mejora de la identidad del barrio, con un espacio más bello, más representativo; el fomento de la interculturalidad, que es un valor que preexistía en el barrio; y la apropiación de un lugar secundario para convertirlo en un símbolo del barrio. Esto se leía en el periódico del barrio como orgullo "El barrio ya tiene una obra de Boa Mistura" (Tetuán 30 días, 2016: 7). Se trata del valor del arte urbano como símbolo de empoderamiento.

En todo caso, como lecciones aprendidas en la construcción del proceso, resulta necesaria la presencia de financiación, aunque no sea muy alta; coordinación, ya que es una intervención aparentemente sencilla, pero que en la práctica ha implicado a un gran número de personas, con trayectorias y tiempos distintos, y de líderes vecinales, como en este caso, Martín Carril Obiols, en representación de la Huerta de Tetuán, con un valor e implicación especial en cuanto a que juega el rol principal de intermediador; sin figuras clave, como en este caso él, resultaría casi imposible poder llevar a cabo este proceso con la legitimidad de hacerse por y para los vecinos e inmigrantes. 
A modo de resumen de las conclusiones se incluye la siguiente tabla (Tabla 2) que trata de destacar los principales aspectos que se han tenido en cuenta tanto en la construcción del proceso como en la intervención mural.

\begin{tabular}{|c|c|c|}
\hline $\begin{array}{l}\text { Categoría } \\
\text { Axiológica }\end{array}$ & $\begin{array}{l}\text { La construcción del proceso } \\
\text { de participación }\end{array}$ & $\begin{array}{l}\text { La intervención mural de Boa } \\
\text { Mistura en Tetuán, Madrid }\end{array}$ \\
\hline PARTICIPACIÓN & $\begin{array}{l}\text { Derecho a la producción del } \\
\text { espacio } \\
\text { Creación de consensos } \\
\text { Gestión para la involucra- } \\
\text { ción de agentes diversos }\end{array}$ & $\begin{array}{l}\text { Creación artística compartida } \\
\text { con amplia participación de } \\
\text { diferentes perfiles } \\
\text { Asociación a otras interven- } \\
\text { ciones previas cercanas como } \\
\text { la Huerta de Tetuán y, en ge- } \\
\text { neral, en Paisaje Tetuán }\end{array}$ \\
\hline CREACIÓN & $\begin{array}{l}\text { Diseño teniendo en cuenta } \\
\text { propuestas de vecinos, ni- } \\
\text { nos y la administración pú- } \\
\text { blica: requerimientos }\end{array}$ & $\begin{array}{l}\text { Formación durante el proceso } \\
\text { de pintura a través del colec- } \\
\text { tivo de artistas Boa Mistura } \\
\text { Cooperación entre colabora- } \\
\text { dores diarios y participantes } \\
\text { esporádicos }\end{array}$ \\
\hline RECREO & Arte urbano participativo & $\begin{array}{l}\text { Mejora del espacio público: } \\
\text { embellecimiento } \\
\text { Propuesta creativa de proxi- } \\
\text { midad }\end{array}$ \\
\hline IDENTIDAD & $\begin{array}{l}\text { Mejora de la autoestima de } \\
\text { los vecinos y de todos los } \\
\text { participantes } \\
\text { Mejora de la identidad del } \\
\text { barrio } \\
\text { Fomento de la interculturali- } \\
\text { dad: valor propio del barrio }\end{array}$ & $\begin{array}{l}\text { Apropiación de un lugar de } \\
\text { periferia interna para conver- } \\
\text { tirlo en un símbolo del barrio } \\
\text { "El barrio ya tiene una obra } \\
\text { de Boa Mistura": el arte ur- } \\
\text { bano como símbolo de empo- } \\
\text { deramiento }\end{array}$ \\
\hline
\end{tabular}

Tabla 2. Elaboración propia de los valores de la construcción del proceso de participación e intervención mural respecto a los valores de participación, creación, recreo e identidad. 


\section{Bibliografía.}

ADVANCED BUILDING SKINS (2017): Conference program Advanced Building Skins 2017. https://abs.green/home/. Acceso 15 de mayo de 2017.

ALGUACIL, Julio (2008): “Espacio público y espacio político. La ciudad como el lugar para las estrategias de participación". Polis. Revista de Universidad Bolivariana de Chile, v.7 n²0; págs.: 199-223.

AlGUACIL, Julio y MONTANÉS, Manuel (2004): “Apostando por la producción del espacio urbano de manera participada", Revista Cuchará y "paso atrás", Volumen: no 8 Páginas, pp. 55 - 72, Acsur - Las Segovias. Citado como 1998 en Alguacil, Julio (2008).

Augé, Marc (1993): Los no lugares: espacios del anonimato. Una antropología de la sobremodernidad. Gedisa, Barcelona.

BOA MistURA (2017a): Projects. http://www.boamistura.com/projects.html. Acceso 10 de abril de 2017.

BOA MisturA (2017b): Cinco cabezas, diez manos, un solo corazón. http://www.boamistura.com/about-us.html. Acceso 10 de abril de 2017.

BORJA, Jordi (2000): “Ciudadanía y espacio público”, en JIMÉNEZ, David. Laberintos urbanos en América Latina. Editorial ABYA-YALA, Serie Pluriminor, Quito, Ecuador.

DeBorD, Guy (2010): La sociedad del espectáculo. Valencia, Pre - Textos.

DelGADO, Manuel (2004): "La no - ciudad como ciudad absoluta", en La arquitectura de la no-ciudad. Pamplona, Cátedra Jorge Oteiza - Universidad Pública de Navarra.

DelGADO JimÉNEZ, Alexandra (2010): “De la participación ciudadana a la gobernanza urbana: transformaciones políticas y territoriales", Boletín $C F+S$, 44, pp. 67-78. Recurso electrónico en línea: http://habitat.aq.upm.es/ boletin/n44/aadel.html. Acceso el 17 de septiembre de 2017.

DEPARTAMENTO de Lenguas Aplicadas y Educación de la Universidad Nebrija (2016): Dossier Altĕritas.

El Viajero (2016): “Un premio al color de Boa Mistura”, El viajero, El País, 21 de marzo de 2016, http://elviajero.elpais.com/elviajero/2016/03/08/album/ 1457457661_201917.html\#1457457661_201917_1457458127. Acceso 10 de abril de 2017. 
FunEs RIVAS, María Jesús y MONFERRER, Jordi (2003): “Perspectivas teóricas y aproximaciones metodológicas al estudio de la participación", En Movimientos sociales: cambio social y participación., editores: FUNES RIVAS, María Jesús y ADELL ARGILÉS, Ramón, Madrid: Universidad Nacional de Educación a Distancia (UNED).

GIDDINGS, Bob, CHARLTON, James y HORNE, Margaret (2011): “Public squares in European city centres". Urban Design international. Vol. 16, 3, pp. 202-212.

IVESON, Kurt (2013): “Cities within the City: Do - It - yourself Urbanism and the Right to the City". International Journal of Urban and Regional Research. Vol. 37.3, pp. 941 -956.

KoOLHAAS, Rem (1995): S, M, L, X. New York: Monacelli Press.

LA AVENTURA DE APRENDER: La huerta de Tetuán, http://laaventuradeaprender. educalab.es/-/la-huerta-de-tetuan. Acceso 10 de abril de 2017.

LABORATORIO URBANO (2004): “Construcción del espacio desde la participación", Boletín $C F+S$, No 32: IAU+S: la Sostenibilidad en el Proyecto Arquitectónico y Urbanístico. Disponible en: http://habitat.aq.upm.es/boletin /n32/nlurb02.html Acceso 15 de julio de 2017.

LYNCH, Kevin (2012): La imagen de la ciudad. Barcelona, Gustavo Gili.

MAlaPARTE, Curzio (1969): La piel. Ediciones G.P. Barcelona, Del original La pelle, Roma-Milano, Aria d'Italia, 1949, pp. 416.

MAX-NefF, Manfred; ElIZALDE, Antonio y Hopenhayn, Martin (2010): “Desarrollo a escala humana: una opción para el futuro", Development Dialogue, número especial, CEPAUR y Fundación Dag Hammarskjöld, Uppsala.

O'CONNOR, Rosin (2017): “Banksy offers free art to people who vote against the Tories", The Independent, http:/www.independent.co.uk/arts-entertainment/ art/news/general-election-2017-banksy-free-print-vote-against-conservativeparty-theresa-may-eu-brexit-a7772341.html. Acceso 10 de junio de 2017.

PRIETO, Eduardo (2011): La arquitectura de la ciudad global. Madrid, Biblioteca Nueva.

Rossi, Aldo (2004): La arquitectura de la ciudad. Barcelona, Gustavo Gili.

RuIz MANTILla, Jesús (2016): "Boa Mistura, el arte de tatuar la ciudad", El País, 10 de junio de 2016, http://cultura.elpais.com/cultura/2016/06/10/ actualidad/1465578586_837322.html. 
SimOes AELBRECHT, Patricia (2016): "Fourth places": the contemporary public settings for informal social interaction among strangers". Journal of Urban Design, pp. 1 -29.

STAUSKIS, Gintaras y ECKARDT, Frank (2011): “Empowering public spaces as catalysers of social interactions in urban communities". Urbanistika ir Architektüra - Town Planning and Architecture. № 35(2), pp. 117 -128.

TETUÁN 30 DíAs (2016): “El colectivo participa en el "Muro de la convivencia”: El arte urbano de Boa Mistura ya tiene su sello en el barrio", Tetuán 30 días, julio- agosto 2016, http://tetuan30dias.com/hemeroteca/t30d-jun16.pdf, página 7. Acceso 12 de junio de 2016.

UNIVERSIDAD NEBRIJA (2016a): “Proyecto Altĕritas: La pintura como herramienta de integración social", http://www.nebrija.com/medios/ rednebrijanoticias/2016/06/30/proyecto-alteritas-la-pintura-como-herramienta-deintegracion-social-2/. Acceso 12 de junio de 2017.

UNIVERSIDAD NEBRIJA (2016b): “Encuentro con el colectivo Boa Mistura en la Universidad Nebrija", http://www.nebrija.com/medios/actualidadnebrija/ 2016/05/17/encuentro-colectivo-boa-mistura-la-universidad-nebrija/. Acceso 12 de junio de 2017. 